\title{
High-Rate Codes with Sublinear-Time Decoding
}

\author{
Swastik Kopparty ${ }^{*}$ \\ Institute for Advanced Study \\ swastik@ias.edu
}

\author{
Shubhangi Saraf \\ MIT \\ shibs@mit.edu
}

\author{
Sergey Yekhanin \\ Microsoft Research \\ yekhanin@microsoft.com
}

\begin{abstract}
Locally decodable codes are error-correcting codes that admit efficient decoding algorithms; any bit of the original message can be recovered by looking at only a small number of locations of a corrupted codeword. The tradeoff between the rate of a code and the locality/efficiency of its decoding algorithms has been well studied, and it has widely been suspected that nontrivial locality must come at the price of low rate. A particular setting of potential interest in practice is codes of constant rate. For such codes, decoding algorithms with locality $O\left(k^{\epsilon}\right)$ were known only for codes of rate $\exp (1 / \epsilon)$, where $k$ is the length of the message. Furthermore, for codes of rate $>1 / 2$, no nontrivial locality has been achieved.

In this paper we construct a new family of locally decodable codes that have very efficient local decoding algorithms, and at the same time have rate approaching 1 . We show that for every $\epsilon>0$ and $\alpha>0$, for infinitely many $k$, there exists a code $C$ which encodes messages of length $k$ with rate $1-\alpha$, and is locally decodable from a constant fraction of errors using $O\left(k^{\epsilon}\right)$ queries and time. The high rate and local decodability are evident even in concrete settings (and not just in asymptotic behavior), giving hope that local decoding techniques may have practical implications.

These codes, which we call multiplicity codes, are based on evaluating high degree multivariate polynomials and their derivatives. Multiplicity codes extend traditional multivariate polynomial based codes; they inherit the local-decodability of these codes, and at the same time achieve better tradeoffs and flexibility in their rate and distance.
\end{abstract}

\footnotetext{
* Part of this work was done while the author was an intern at Microsoft Research Silicon Valley. The author is supported by National Science Foundation Grant No. DMS-0835373.

${ }^{\dagger}$ Part of this work was done while the author was an intern at Microsoft Research Silicon Valley.
}

Permission to make digital or hard copies of all or part of this work for personal or classroom use is granted without fee provided that copies are not made or distributed for profit or commercial advantage and that copies bear this notice and the full citation on the first page. To copy otherwise, to republish, to post on servers or to redistribute to lists, requires prior specific permission and/or a fee.

STOC'11, June 6-8, 2011, San Jose, California, USA.

Copyright 2011 ACM 978-1-4503-0691-1/11/06 ...\$10.00.

\section{Categories and Subject Descriptors}

E.4 [Data]: Coding and Information Theory

\section{General Terms}

Algorithms, Theory

\section{Keywords}

Error-correcting codes, Sublinear-time algorithms, locally decodable codes, polynomials, derivatives, multiplicity codes

\section{INTRODUCTION}

Classical error-correcting codes allow one to encode a $k$ bit message $\mathbf{x}$ into an $n$-bit codeword $C(\mathbf{x})$, in such a way that $\mathbf{x}$ can still be recovered even if $C(\mathbf{x})$ gets corrupted in a number of coordinates. The traditional way to recover information about $\mathbf{x}$ given access to a corrupted version of $C(\mathbf{x})$ is to run a decoder for $C$, which would read and process the entire corrupted codeword, and then recover the entire original message $\mathbf{x}$. Suppose that one is only interested in recovering a single bit or a few bits of $\mathbf{x}$. In this case, codes with more efficient decoding schemes are possible, allowing one to read only a small number of code positions. Such codes are known as Locally Decodable Codes (LDCs). Locally decodable codes allow reconstruction of an arbitrary bit $\mathbf{x}_{i}$, by looking only at $t \ll k$ randomly chosen coordinates of (a possibly corrupted) $C(\mathbf{x})$.

The main parameters of a locally decodable code that measure its utility are the codeword length $n$ (as a function of the message length $k$ ) and the query complexity of local decoding. The length measures the amount of redundancy that is introduced into the message by the encoder. The query complexity counts the number of bits that need to be read from a (corrupted) codeword in order to recover a single bit of the message. Ideally, one would like to have both of these parameters as small as possible. One however cannot minimize the codeword length and the query complexity simultaneously; there is a trade-off. On one end of the spectrum we have LDCs with the codeword length close to the message length, decodable with somewhat large query complexity. Such codes are useful for data storage and transmission. On the other end we have LDCs where the query complexity is a small constant but the codeword length is large compared to the message length. Such codes find applications in complexity theory and cryptography. The true shape of the trade-off between the codeword length and the query complexity of LDCs is not known. Determining it is 
a major open problem (see [41] for a recent survey of the LDC literature).

While most prior work focuses on the low query (and even constant query) regime, in this work we will look at the other extreme and consider the setting of locally decodable codes with very low redundancy, which may be of even greater practical interest. More precisely, we will be interested in minimizing the query complexity of local decoding for codes of large rate (defined as the ratio $k / n$, where the code encodes $k$ bits into $n$ bits). For codes of rate $>1 / 2$, it was unknown how to get any nontrivial local decoding whatsoever. For smaller rates, it was known how to construct codes (in fact, the classical Reed-Muller codes based on evaluating multivariate polynomials have this property) which admit local decoding with $O\left(k^{\epsilon}\right)$ queries and time, at the cost of reducing the rate to $\epsilon^{\Omega(1 / \epsilon)}$. In practical applications of coding theory to data storage and transmission, the rate of encoding has always been paramount; using codes of very small rate translates into increasing the storage required or transmission time manifold, and is unacceptable for most applications.

In this paper, we introduce a new and natural family of locally decodable codes, which achieve high rates while admitting local decoding with low query complexity. These codes, which we call multiplicity codes, are based on evaluating multivariate polynomials and their derivatives. They inherit the local-decodability of the traditional multivariate polynomial codes, while achieving better tradeoffs and flexibility in the rate and minimum distance. Using multiplicity codes, we prove (see Theorem 4) that it is possible to have codes that simultaneously have (a) rate approaching 1 , and (b) allow for local decoding with arbitrary polynomiallysmall time and query complexity.

Main Theorem (informal): For every $\epsilon>0, \alpha>0$, and for infinitely many $k$, there exists a code which encodes $k$-bit messages with rate $1-\alpha$, and is locally decodable from some constant fraction of errors using $O\left(k^{\epsilon}\right)$ time and queries.

\subsection{Previous work on locally decodable codes}

The first examples of local decoding algorithms for appeared implicitly in the coding theory community a long time ago, starting with Reed's "majority-logic decoder" for binary Reed-Muller codes [29]. In theoretical computer science, locally decodable codes (and in particular, locally decodable codes based on multivariate polynomials) have played an important part (again implicitly) in the Proof-Checking Revolution of the early 90 s $[7,24,25,33,4,5,2,1]$ as well as in other fundamental results in complexity theory $[6,20$, $3,35,32]$.

Locally decodable codes were first formally defined in $[4$, 22] (see also [35]). Since then, the quest for understanding locally decodable codes has generated many developments. Most of the previous work on LDCs has focussed on local decoding with a constant number of queries. For a long time, it was generally believed that for decoding with constantly many queries, a $k$ bit message must be encoded into at least $\exp \left(k^{\alpha}\right)$ bits, for constant $\alpha>0$. Recently, in a surprising sequence of works $[40,28,14,21,12,8,9]$ this was shown to be soundly false; today we know constant query locally decodable codes which encode $k$ bits into as few as $\exp \left(\exp \left(\log ^{\alpha}(k)\right)\right)$ bits for constant $\alpha>0$.

There has also been considerable work $[22,23,15,36,37$,
$10,26]$ on the problem of proving lower bounds on the length of locally decodable codes. In particular, it is known [22] that for codes of constant rate, local decoding requires at least $\Omega(\log k)$ queries. For codes locally decodable with $\omega(\log k)$ queries, no nontrivial lower bound on the length on the code is known. For error-correction with $O\left(k^{\epsilon}\right)$ queries, Dvir [11] recently conjectured a lower bound on the length of some closely related objects called locally self-correctable codes. Precisely, the conjecture of [11] states that for every field $\mathbb{F}$, there exist positive constants $\alpha$ and $\epsilon$ such that there are no linear codes over $\mathbb{F}$ of length $n$, rate $1-\alpha$ and locally self-correctable with query complexity $O\left(n^{\epsilon}\right)$ from a certain sub-constant fraction of errors. Dvir [11] then showed that establishing this conjecture would yield progress on some well-known open questions in arithmetic circuit complexity.

Our results refute Dvir's conjecture over finite fields; using multiplicity codes, we show that for arbitrary $\alpha, \epsilon>0$, for every finite field $\mathbb{F}$, for infinitely many $n$, there is a linear code over $\mathbb{F}$ of length $n$ with rate $1-\alpha$, which is locally self-correctable from even a constant fraction of errors with $O\left(n^{\epsilon}\right)$ queries ${ }^{1}$.

\subsection{Multiplicity codes}

We now give a quick introduction to multiplicity codes and demonstrate the principles on which they are based.

To minimize extraneous factors and for ease of exposition, in this subsection we will deal with the problem of constructing "locally self-correctable codes" over "large alphabets", which we now define. We have a set $\Sigma$ (the "alphabet"), and we want to construct a subset $\mathcal{C}$ (the "code") of $\Sigma^{n}$, of size $|\Sigma|^{k}$ (we call $k$ the "message length"), with the following local self-correction property: given access to any $r \in \Sigma^{n}$ which is close to some codeword $c \in \mathcal{C}$, and given $i \in[n]$, it is possible to make few queries to the coordinates of $r$, and with high probability output $c_{i}$. The goal is to construct such a subset $\mathcal{C}$ with rate $k / n$ large. Note that this differs from the notion of locally decodable code in that we seek to recover a coordinate of the nearby codeword $c$, not of the original message which encodes to $c$. We also do not require that $\Sigma$ has size 2, which is what the Main Theorem mentioned earlier refers to. Translating from local self-correctability over large alphabets to local decodability over small alphabets is a standard transformation.

Our plan is as follows. We will first recall an example of the classical Reed-Muller codes based on bivariate polynomials and why it is locally self-correctable. We will then introduce the simplest example of a multiplicity code based on bivariate polynomials, which has improved rate, and see how to locally self-correct it with essentially the same query complexity. Finally, we mention how general multiplicity codes are defined and some of the ideas that go into locally self-correcting them.

Bivariate Reed-Muller codes: Let $q$ be a prime power, let $\delta>0$ and let $d=(1-\delta) q$. The Reed Muller code of degree $d$ bivariate polynomials over $\mathbb{F}_{q}$ (the finite field of cardinality $q$ ) is the code defined as follows. The coordinates of the code are indexed by elements of $\mathbb{F}_{q}^{2}$, and so $n=q^{2}$. The codewords are indexed by bivariate polynomials of de-

\footnotetext{
${ }^{1}[11]$ contains two conjectures; which are called the "strong conjecture" and the "weak conjecture". We refute only the strong conjecture. The weak conjecture, which has weaker implications for questions related to arithmetic circuit complexity, remains open.
} 
gree at most $d$ over $\mathbb{F}_{q}$. The codeword corresponding the polynomial $P(X, Y)$ is the vector

$$
C(P)=\langle P(\mathbf{a})\rangle_{(\mathbf{a}) \in \mathbb{F}_{q}^{2}} \in \mathbb{F}_{q}^{q^{2}} .
$$

Because two distinct polynomials of degree at most $d$ can agree on at most $d / q$-fraction of the points in $\mathbb{F}_{q}^{2}$, this code has distance $\delta=1-d / q$. Any polynomial of degree at most $d$ is specified by one coefficient for each of the $\left(\begin{array}{c}d+1 \\ 2\end{array}\right)$ monomials, and so the message length $k=\left(\begin{array}{c}d+1 \\ 2\end{array}\right)$. Thus the rate of this code is $\left(\begin{array}{c}d+1 \\ 2\end{array}\right) / q^{2} \approx(1-\delta)^{2} / 2$. Notice that this code cannot have rate more than $1 / 2$.

Local Self-Correction of Reed-Muller codes: Given a received word $r \in\left(\mathbb{F}_{q}\right)^{q^{2}}$ such that $r$ is close in Hamming distance to the codeword corresponding to $P(X, Y)$, let us recall how the classical local self-correction algorithm works. Given a coordinate $\mathbf{a} \in \mathbb{F}_{q}^{2}$, we want to recover the "corrected" symbol at coordinate a, namely $P(\mathbf{a})$. The algorithm picks a random direction $\mathbf{b} \in \mathbb{F}_{q}^{2}$ and looks at the restriction of $r$ to coordinates in the line $L=\left\{\mathbf{a}+\mathbf{b} t \mid t \in \mathbb{F}_{q}\right\}$. With high probability over the choice of $\mathbf{b}, r$ and $C(P)$ will agree on many positions of $L$. Now $\left.C(P)\right|_{L}$ is simply the vector consisting of evaluations of the univariate polynomial $Q(T)=P(\mathbf{a}+\mathbf{b} T) \in \mathbb{F}_{q}[T]$, which is of degree $\leq d$. Thus $\left.r\right|_{L}$ gives us $q$ "noisy" evaluations of a polynomial $Q(T)$ of degree $\leq(1-\delta) \cdot q$; this enables us to recover $Q(T)$. Evaluating $Q(T)$ at $T=0$ gives us $P(\mathbf{a})$, as desired. Notice that this decoding algorithm makes $q$ queries, which is $O\left(k^{1 / 2}\right)$.

Bivariate Multiplicity Codes: We now introduce the simplest example of multiplicity codes, which already achieves a better rate than the Reed-Muller code above, while being locally self-correctable with only a constant factor more queries.

Let $q$ be a prime power, let $\delta>0$ and let $d=2(1-\delta) q$ (which is twice what it was in the Reed-Muller example). The multiplicity code of order $\mathbf{2}$ evaluations of degree $d$ bivariate polynomials over $\mathbb{F}_{q}$ is the code defined as follows. As before, the coordinates are indexed by $\mathbb{F}_{q}^{2}$ (so $n=q^{2}$ ) and the codewords are indexed by bivariate polynomials of degree at most $d$ over $\mathbb{F}_{q}$. However the alphabet will now be $\mathbb{F}_{q}^{3}$. The codeword corresponding the polynomial $P(X, Y)$ is the vector

$$
C(P)=\left\langle\left(P(\mathbf{a}), \frac{\partial P}{\partial X}(\mathbf{a}), \frac{\partial P}{\partial Y}(\mathbf{a})\right)\right\rangle_{(\mathbf{a}) \in \mathbb{F}_{q}^{2}} \in\left(\mathbb{F}_{q}^{3}\right)^{q^{2}} .
$$

In words, the a coordinate consists of the evaluation of $P$ and its partial derivatives $\frac{\partial P}{\partial X}$ and $\frac{\partial P}{\partial Y}$ at a. Because two distinct polynomials of degree at most $d$ can agree with multiplicity 2 on at most $d / 2 q$-fraction of the points in $\mathbb{F}_{q}^{2}$, this code has distance $\delta=1-d / 2 q$. Since the alphabet size is now $q^{3}$, the message length $k$ equals the number of $q^{3}$-ary symbols required to specify a polynomial of degree at most $d$; this is clearly $\left(\begin{array}{c}d+1 \\ 2\end{array}\right) / 3$. Thus the rate of this code is $\left(\left(\begin{array}{c}d+1 \\ 2\end{array}\right) / 3\right) / q^{2} \approx 2(1-\delta)^{2} / 3$

Summarizing the differences between this multiplicity code with the Reed-Muller code described earlier: (a) instead of polynomials of degree $(1-\delta) q$, we consider polynomials of degree double of that, (b) instead of evaluating the polynomials, we take their "order-2" evaluation. This yields a code with the same distance, while the rate improved from $<1 / 2$ to nearly $2 / 3$.

Local Self-Correction of Multiplicity codes: Given a received word $r \in\left(\mathbb{F}_{q}^{3}\right)^{q^{2}}$ such that $r$ is close in Hamming distance to the codeword corresponding to $P(X, Y)$, we will show how to locally self-correct. Given a point a $\in \mathbb{F}_{q}^{2}$, we want to recover the "corrected" symbol at coordinate $\mathbf{a}$, namely $\left(P(\mathbf{a}), \frac{\partial P}{\partial X}(\mathbf{a}), \frac{\partial P}{\partial Y}(\mathbf{a})\right)$. Again, the algorithm picks a random direction $\mathbf{b}=\left(b_{1}, b_{2}\right) \in \mathbb{F}_{q}^{2}$ and looks at the restriction of $r$ to coordinates in the line $L=\left\{\mathbf{a}+\mathbf{b} t \mid t \in \mathbb{F}_{q}\right\}$. With high probability over the choice of $\mathbf{b}$, we will have that $\left.r\right|_{L}$ and $\left.C(P)\right|_{L}$ agree in many locations. Our intermediate goal will be to recover the univariate polynomial ${ }^{2}$ $Q(T)=P(\mathbf{a}+\mathbf{b} T)$. The important observation is that for every $t \in \mathbb{F}_{q}$, the $\mathbf{a}+\mathbf{b} t$ coordinate of $C(P)$ completely determines both the value and the 1st derivative of the univariate polynomial $Q(T)$ at the point $t$; indeed, by the chain rule we have:

$\left(Q(t), \frac{\partial Q}{\partial T}(t)\right)=\left(P(\mathbf{a}+\mathbf{b} t), b_{1} \frac{\partial P}{\partial X}(\mathbf{a}+\mathbf{b} t)+b_{2} \frac{\partial P}{\partial Y}(\mathbf{a}+\mathbf{b} t)\right)$.

Thus our knowledge of $\left.r\right|_{L}$ gives us access to $q$ "noisy" evaluations of the polynomial $Q(T)$ and its derivative $\frac{\partial Q}{\partial T}(T)$, where $Q(T)$ is of degree $\leq 2(1-\delta) q$. It turns out that this is enough to recover the polynomial $Q(T)$. Evaluating $Q(T)$ at $T=0$ gives us $P(\mathbf{a})$. Evaluating the derivative $\frac{\partial Q}{\partial T}(T)$ at $T=0$ gives us the directional derivative of $P$ at $\mathbf{a}$ in the direction $\mathbf{b}$ (which equals $b_{1} \frac{\partial P}{\partial X}(\mathbf{a})+b_{2} \frac{\partial P}{\partial Y}(\mathbf{a})$ ). We have clearly progressed towards our goal of computing the tuple $\left(P(\mathbf{a}), \frac{\partial P}{\partial X}(\mathbf{a}), \frac{\partial P}{\partial Y}(\mathbf{a})\right)$, but we are not yet there. The final observation is that if we pick another direction $\mathbf{b}^{\prime}$, and repeat the above process to recover the directional derivative of $P$ at $\mathbf{a}$ in direction $\mathbf{b}^{\prime}$, then the two directional derivatives of $P$ at $\mathbf{a}$ in directions $\mathbf{b}, \mathbf{b}^{\prime}$ together suffice to recover $\frac{\partial P}{\partial X}(\mathbf{a})$ and $\frac{\partial P}{\partial Y}(\mathbf{a})$, as desired. This algorithm makes $2 q$ queries, which is $O\left(k^{1 / 2}\right)$.

General Multiplicity codes: The basic example of a multiplicity code above already achieves rate $R>1 / 2$ while allowing local decoding with sublinear query complexity (which was not known before). To get codes of rate approaching 1 , we modify the above example by considering evaluations of all derivatives of $P$ up to an even higher order. In order to locally recover the higher-order derivatives of $P$ at a point $\mathbf{a}$, the decoding algorithm will pick many random lines passing through a, try to recover the restriction of $P$ to those lines, and combine all these recovered univariate polynomials in a certain way. To reduce the query complexity to $O\left(k^{\epsilon}\right)$ for small $\epsilon$, we modify the above example by considering multivariate polynomials in a larger number of variables $m$. The local decoding algorithm for this case, in order to locally recover at a point $\mathbf{a} \in \mathbb{F}_{q}^{m}$, decodes by picking random lines passing through a; the reduced query complexity occurs because lines (with only $q$ points) are now much smaller relative to a higher dimensional space $\mathbb{F}_{q}^{m}$. Increasing both the maximum order of derivative taken and the number of variables simultaneously yields multiplicity codes with the desired rate and local decodability.

In Section 4, we present our local self-correction algorithm, which implements the plan outlined above, along with an extra "robustification" so that the fraction of errors which can be recovered from is a constant fraction of the distance of the code. We also show how the algorithm can be made to run in sublinear time (almost as small as the query complexity).

\footnotetext{
${ }^{2}$ Unlike in the Reed-Muller case, here there is a distinction between recovering $Q(T)$ and recovering $\left.C(P)\right|_{L}$. It turns out that recovering $\left.C(P)\right|_{L}$ given only $\left.r\right|_{L}$ is impossible.
} 
Applications of derivatives and multiplicities: The notions of derivative and multiplicity have played an important role in several prior works in coding theory and theoretical computer science. The "method of multiplicities" is a powerful combinatorial/algorithmic technique which has been developed and used in a number of contexts in recent years $[18,27,16,31,13]$. It is a method for analyzing subsets of $\mathbb{F}_{q}^{m}$ by interpolating a polynomial that vanishes at each point of that subset with high multiplicity; this often yields a strengthening of the "polynomial method", which would analyze such a subset by interpolating a polynomial that simply vanishes at each point of that subset. Rozenbloom and Tsfasman [30] consider extensions of Reed-Solomon codes where the polynomial is evaluated together with its derivatives (basically, univariate multiplicity codes) to obtain codes for some metrics generalizing the usual Hamming metric. Xing [39] considers the space of differentials on an algebraic curve to prove the existence of error-correcting codes above the Tsfasman-VladutZink bound. Woodruff and Yekhanin [38] use evaluations of polynomials and their derivatives to construct private information retrieval schemes with improved communication complexity. Multiplicity codes add to this body of work, which follows the general theme that wherever polynomials and their zeroes are useful, also considering their derivatives and high-multiplicity zeroes can be even more useful ${ }^{3}$.

\section{Organization of this paper:}

In the next section, we state our main theorems on the existence of locally decodable/self-correctable codes. In Section 3, we formally define multiplicity codes, calculate their rate and distance, state the theorem implying their local decodability, and show how they imply the main theorems from the previous section. In Section 4 we give our local self-correction algorithms for multiplicity codes. Section 5 contains some concluding remarks.

\section{MAIN RESULTS ON THE EXISTENCE OF LOCALLY DECODABLE CODES}

In this section, we state our main results on the existence of locally decodable codes with rate approaching 1 . We begin with some standard definitions.

For a set $\Sigma$ and two vectors $c, c^{\prime} \in \Sigma^{n}$, we define their relative Hamming distance $\Delta\left(c, c^{\prime}\right)$ to be the fraction of coordinates where they differ: $\Delta\left(c, c^{\prime}\right)=\operatorname{Pr}_{i \in[n]}\left[c_{i} \neq c_{i}^{\prime}\right]$.

An error-correcting code of length $n$ over the alphabet $\Sigma$ is a subset $\mathcal{C} \subseteq \Sigma^{n}$. The rate of $\mathcal{C}$ is the defined to be $\frac{\log |\mathcal{C}|}{n \log |\Sigma|}$. The (minimum) distance of $\mathcal{C}$ is defined to be the smallest $\delta>0$ such that for every distinct $c_{1}, c_{2} \in \mathcal{C}$, we have $\Delta\left(c_{1}, c_{2}\right) \geq \delta$.

For $q$ a prime power, let $\mathbb{F}_{q}$ denote the finite field on $q$ elements. If $\Sigma=\mathbb{F}_{q}$, then a code $\mathcal{C}$ over the alphabet $\Sigma$ is called a linear code if $\mathcal{C}$ is a $\mathbb{F}_{q}$-linear subspace of $\Sigma^{n}$.

We now define locally self-correctable codes and locally decodable codes. For an algorithm $\mathrm{A}$ and a string $r$, we will use $A^{r}$ to represent the situation where $A$ is given query access to $r$.

Definition 1 (Locally Self-correctable Code). $A$ code $\mathcal{C} \subseteq \Sigma^{n}$ is said to be locally self-correctable from $\delta^{\prime}$ - fraction errors with $t$ queries if there is a randomized algorithm A, such that:

- Self-correction: Whenever $c \in \mathcal{C}$ and $r \in \Sigma^{n}$ are such that $\Delta(r, c)<\delta^{\prime}$, then for each $i \in[n]$,

$$
\operatorname{Pr}\left[\mathrm{A}^{r}(i)=c_{i}\right] \geq 2 / 3 \text {. }
$$

- Query complexity $t: \mathrm{A}^{r}(i)$ always makes at most $t$ queries to $r$.

Definition 2 (Locally Decodable Code). Let $\mathcal{C} \subseteq$ $\Sigma^{n}$ be a code with $|\mathcal{C}|=|\Sigma|^{k}$. Let $E: \Sigma^{k} \rightarrow \mathcal{C}$ be a bijection (we refer to $E$ as the encoding map for $\mathcal{C}$; note that $k / n$ equals the rate of the code $\mathcal{C})$. We say that $(\mathcal{C}, E)$ is locally decodable from $\delta^{\prime}$-fraction errors with $t$ queries if there is a randomized algorithm $\mathrm{A}$, such that:

- Decoding: Whenever $x \in \Sigma^{k}$ and $r \in \Sigma^{n}$ are such that $\Delta(r, E(x))<\delta^{\prime}$, then for each $i \in[k]$,

$$
\operatorname{Pr}\left[\mathrm{A}^{r}(i)=x_{i}\right] \geq 2 / 3 \text {. }
$$

- Query complexity $t: \mathrm{A}^{r}(i)$ always makes at most $t$ queries to $r$.

Suppose $\Sigma=\mathbb{F}_{q}$, and that $\mathcal{C}$ is a linear code over $\Sigma$. By simple linear algebra, it follows that there is an encoding function $E$ such that for each $x \in \Sigma^{k}$ and each $i \in[k]$ there is a $j \in[n]$, such that $E(x)_{j}=x_{i}$. This implies that if $\mathcal{C}$ is locally self-correctable (from some fraction of errors with some query complexity), then $(\mathcal{C}, E)$ is locally decodable (from the same fraction of errors and with the same query complexity). This will allow us to focus on constructing linear codes which are locally self-correctable.

We now state the two main theorems, which assert the existence of locally self-correctable codes with improved rate and query complexity. The first theorem, which does this over a large alphabet (and does not give a linear code), will be a direct consequence of what we show about multiplicity codes in the next section.

THEOREM 3. (LOCALLY SELF-CORRECTABLE CODES OVER LARGE ALPHABETS) For every $0<\epsilon, \alpha<1$, for infinitely many $n$, there is a code $C$ over an alphabet $\Sigma$, with $|\Sigma| \leq$ $n^{O(1)}$, such that $C$ has length $n$, rate at least $1-\alpha$, distance $\delta \geq \epsilon \alpha / 2$, and is locally self-correctable from $\delta / 10$-fraction errors with $O\left(n^{\epsilon}\right)$ queries.

The next theorem is the analogue of theorem 3 for small alphabets (and gives linear codes). These codes are obtained by simply concatenating multiplicity codes with suitable good linear codes over the small alphabet. In particular, this shows the existence of locally decodable codes with similar parameters.

THEOREM 4. (LOCALLY SELF-CORRECTABLE CODES OVER SMALL ALPHABETS) Let $p$ be a prime power. For every $\epsilon, \alpha>0$, there exists $\delta>0$, such that for infinitely many $n$, there is a linear code $\mathcal{C}$ over the alphabet $\Sigma=\mathbb{F}_{p}$, such that $\mathcal{C}$ has length $n$, rate at least $1-\alpha$, distance at least $\delta$, and is locally self-correctable from $\delta / 20$-fraction errors with $O\left(n^{\epsilon}\right)$ queries.

REMARK The codes in both the above theorems are efficiently constructible. Furthermore, both the local selfcorrection algorithms can be made to run in time $O\left(n^{2 \epsilon}\right)$.

The proofs of the theorems above appear in Section 3.3. 


\section{MULTIPLICITY CODES}

In this section we formally define multiplicity codes, calculate their rate and distance, and state the main theorem implying their decodability. We then show how multiplicity codes imply the main theorems of the previous section.

First, we recall some preliminaries on derivatives and multiplicities. We will define our codes using the Hasse derivative, which is a variant of the usual notion of derivative of a polynomial, and is more suitable for use in fields of small characteristic.

\subsection{Derivatives and multiplicities}

We start with some notation. We use $[m]$ to denote the set $\{1, \ldots, m\}$. For a vector $\mathbf{i}=\left\langle i_{1}, \ldots, i_{m}\right\rangle$ of non-negative integers, its weight, denoted wt(i), equals $\sum_{j=1}^{m} i_{j}$.

For a field $\mathbb{F}$, let $\mathbb{F}\left[X_{1}, \ldots, X_{m}\right]=\mathbb{F}[\mathbf{X}]$ be the ring of polynomials in the variables $X_{1}, \ldots, X_{m}$ with coefficients in $\mathbb{F}$.

For a vector of non-negative integers $\mathbf{i}=\left\langle i_{1}, \ldots, i_{m}\right\rangle$, let $\mathbf{X}^{\mathbf{i}}$ denote the monomial $\prod_{j=1}^{m} X_{j}^{i_{j}} \in \mathbb{F}[\mathbf{X}]$. Note that the (total) degree of this monomial equals wt(i).

Definition 5 ((Hasse) Derivative). For $P(\mathbf{X}) \in \mathbb{F}[\mathbf{X}]$ and non-negative vector $\mathbf{i}$, the $\mathbf{i}$ th (Hasse) derivative of $P$, denoted $P^{(\mathbf{i})}(\mathbf{X})$, is the coefficient of $\mathbf{Z}^{\mathbf{i}}$ in the polynomial $\tilde{P}(\mathbf{X}, \mathbf{Z}) \stackrel{\text { def }}{=} P(\mathbf{X}+\mathbf{Z}) \in \mathbb{F}[\mathbf{X}, \mathbf{Z}]$.

Thus,

$$
P(\mathbf{X}+\mathbf{Z})=\sum_{\mathbf{i}} P^{(\mathbf{i})}(\mathbf{X}) \mathbf{Z}^{\mathbf{i}}
$$

Observe that for all $P, Q \in \mathbb{F}[X]$, and $\lambda \in \mathbb{F}$,

$$
(\lambda P)^{(\mathbf{i})}(\mathbf{X})=\lambda P^{(\mathbf{i})}(\mathbf{X}) \text { and } \quad \mathrm{P}^{(\mathbf{i})}(\mathbf{X})+\mathrm{Q}^{(\mathbf{i})}(\mathbf{X})=(\mathrm{P}+\mathrm{Q})^{(\mathbf{i})}(\mathbf{X})
$$

We are now ready to define the notion of the (zero-)multiplicity of a polynomial at any given point.

Definition 6 (Multiplicity). For $P(\mathbf{X}) \in \mathbb{F}[\mathbf{X}]$ and $\mathbf{a} \in \mathbb{F}^{m}$, the multiplicity of $P$ at $\mathbf{a} \in \mathbb{F}^{m}$, denoted mult $(P, \mathbf{a})$, is the largest integer $M$ such that for every non-negative vector $\mathbf{i}$ with $\operatorname{wt}(\mathbf{i})<M$, we have $P^{(\mathbf{i})}(\mathbf{a})=0$ (if $M$ may be taken arbitrarily large, we set $\operatorname{mult}(P, \mathbf{a})=\infty)$.

Note that $\operatorname{mult}(P, \mathbf{a}) \geq 0$ for every a.

The main technical fact we will need about derivatives and multiplicities is a bound on the number of points that a low-degree polynomial can vanish on with high multiplicity. We state this lemma below. For an elementary proof in our notation, see [13].

Lemma 7. Let $P \in \mathbb{F}[\mathbf{X}]$ be a nonzero polynomial of total degree at most $d$. Then for any finite $S \subseteq \mathbb{F}$,

$$
\sum_{\mathbf{a} \in S^{m}} \operatorname{mult}(P, \mathbf{a}) \leq d \cdot|S|^{m-1} \text {. }
$$

In particular, for any integer $s>0$,

$$
\underset{\mathbf{a} \in S^{m}}{\operatorname{Pr}}[\operatorname{mult}(P, \mathbf{a}) \geq s] \leq \frac{d}{s|S|} .
$$

\subsection{The definition of multiplicity codes}

We now come to the definition of multiplicity codes.
Definition 8 (Multiplicity CODE). Let $s, d, m$ be nonnegative integers and let $q$ be a prime power. Let $\Sigma=$ $\mathbb{F}_{q}^{\left(\begin{array}{c}m+s-1 \\ m\end{array}\right)}=\mathbb{F}_{q}^{\{\mathbf{i}: \mathrm{wt}(\mathbf{i})<s\}}$. For $P\left(X_{1}, \ldots, X_{m}\right) \in \mathbb{F}_{q}\left[X_{1}, \ldots, X_{m}\right]$, we define the order $s$ evaluation of $P$ at $\mathbf{a}$, denoted $P^{(<s)}(\mathbf{a})$, to be the vector $\left\langle P^{(\mathbf{i})}(\mathbf{a})\right\rangle_{\mathrm{wt}(\mathbf{i})<s} \in \Sigma$.

We define the multiplicity code of order $s$ evaluations of degree $d$ polynomials in $m$ variables over $\mathbb{F}_{q}$ as follows. The code is over the alphabet $\Sigma$, and has length $q^{m}$ (where the coordinates are indexed by elements of $\left.\mathbb{F}_{q}^{m}\right)$. For each polynomial $P(\mathbf{X}) \in \mathbb{F}_{q}\left[X_{1}, \ldots, X_{m}\right]$ with $\operatorname{deg}(P) \leq d$, there is a codeword in $\mathcal{C}$ given by:

$$
\operatorname{Enc}_{s, d, m, q}(P)=\left\langle P^{(<s)}(\mathbf{a})\right\rangle_{\mathbf{a} \in \mathbb{F}_{q}^{m}} \in(\Sigma)^{q^{m}} .
$$

In the next lemma, we calculate the rate and distance of multiplicity codes. The striking feature of the behavior here is that if we keep the distance $\delta$ fixed and let the multiplicity parameter $s$ grow, the rate of these codes improves (and approaches $\left.(1-\delta)^{m}\right)$.

LEMma 9 (RATE AND DistANCE OF MULTiPlicity CODES). Let $\mathcal{C}$ be the multiplicity code of order s evaluations of degree $d$ polynomials in $m$ variables over $\mathbb{F}_{q}$. Then $\mathcal{C}$ has distance $\delta=1-\frac{d}{s q}$ and rate $\frac{\left(\begin{array}{c}d+m \\ m\end{array}\right)}{\left(\begin{array}{c}s+m \\ m\end{array}-1\right) q^{m}}$, which is at least

$$
\left(\frac{s}{m+s}\right)^{m} \cdot\left(\frac{d}{s q}\right)^{m} \geq\left(1-\frac{m^{2}}{s}\right)(1-\delta)^{m}
$$

Proof. The alphabet size equals $q^{\left(\frac{m+s-1}{m}\right)}$. The length equals $q^{m}$.

To calculate the distance, consider any two codewords $c_{1}=\operatorname{Enc}_{s, d, m, q}\left(P_{1}\right), c_{2}=\operatorname{Enc}_{s, d, m, q}\left(P_{2}\right)$, where $P_{1} \neq P_{2}$. For any coordinate $\mathbf{a} \in \mathbb{F}_{q}^{m}$ where the codewords $c_{1}, c_{2}$ agree (i.e., $\left.\left(c_{1}\right)_{\mathbf{a}}=\left(c_{2}\right) \mathbf{a}\right)$, we have that $P_{1}^{(<s)}(\mathbf{a})=P_{2}^{(<s)}(\mathbf{a})$. Thus for any such $\mathbf{a}$, we have $\left(P_{1}-P_{2}\right)^{(\mathbf{i})}(\mathbf{a})=0$ for each i with wt $(\mathbf{i})<s$, and hence $\operatorname{mult}\left(P_{1}-P_{2}, \mathbf{a}\right) \geq s$. From the bound on the number of high-multiplicity zeroes of multivariate polynomials, Lemma 7 , the fraction of $\mathbf{a} \in \mathbb{F}_{q}^{m}$ on which this can happen is at most $\frac{d}{s q}$. The minimum distance $\delta$ of the multiplicity code is therefore at least $\delta=1-\frac{d}{s q}$.

A codeword is specified by giving coefficients to each of the monomials of degree at most $d$. Thus the number of codewords equals $q^{\left(\begin{array}{c}d+m \\ m\end{array}\right)}$. Thus the rate equals

$$
\begin{aligned}
\frac{\left(\begin{array}{c}
d+m \\
m
\end{array}\right)}{\left(\begin{array}{c}
s+m-1 \\
m
\end{array}\right) q^{m}} & =\frac{\prod_{j=0}^{m-1}(d+m-j)}{\prod_{j=1}^{m}((s+m-j) q)} \\
& \geq\left(\frac{1}{1+\frac{m}{s}}\right)^{m}\left(\frac{d}{s q}\right)^{m} \\
& \geq\left(1-\frac{m^{2}}{s}\right)(1-\delta)^{m} .
\end{aligned}
$$

The next theorem, which will be the focus of the rest of this paper, shows that multiplicity codes are locally selfcorrectable.

ThEOREM 10. (MUlTiPlicity CODES ARE LOCALLY SELFCORRECTABLE) Let $\mathcal{C}$ be the multiplicity code of order $s$ evaluations of degree $d$ polynomials in $m$ variables over $\mathbb{F}_{q}$. Let $\delta=1-d / s q$ be the distance of $\mathcal{C}$. Suppose $q \geq \max \left\{10 \mathrm{~m}, \frac{d+6}{s}, 5(s+\right.$ $1)\}$. Then $\mathcal{C}$ is locally self-correctable from $\frac{\delta}{10}$-fraction errors with $O(s)^{m} \cdot q$-queries. 
The proof of this theorem appears in Section 4.2. In Section 4.3 , we will show that the local self-corrector can also be made to run very efficiently, in time $O(s)^{m} \cdot q^{O(1)}$.

Multiplicity codes can also be locally decoded with a factor $\exp (m+s)$-increase in the query complexity, for a suitable choice of encoding function (even though multiplicity codes are not linear). We omit the details.

\subsection{Proof of the main theorems}

We now show how to instantiate multiplicity codes to prove our main theorems on the existence of locally selfcorrectable codes with improved rate and query-complexity (assuming Theorem 10).

Proof of TheOrem 3: Recall that we are trying to construct, for ever $0<\epsilon, \alpha<1$, for infinitely many $n$, a code over an alphabet of size $n^{O(1)}$, with length $n$, rate $\geq 1-\alpha$, distance $\delta \geq \epsilon \alpha / 2$, and locally self-correctable with $O\left(n^{\epsilon}\right)$ queries from $\delta / 10$-fraction errors.

Pick $m=\lceil 1 / \epsilon\rceil$. For every large enough prime power $q$, we will construct such a code with $n=q^{m}$. Pick $s$ so that

$$
1-\frac{m^{2}}{s}>\frac{1-\alpha}{(1-\delta)^{m}}
$$

(this can be done with $s=O\left(m^{2}\right)$ ). Observe that $m$ and $s$ are constants. Let $d=(1-\delta) \cdot s \cdot q$. Observe that for all $\alpha, \epsilon, 1-\alpha<(1-\epsilon \alpha / 2)^{2 / \epsilon}<(1-\epsilon \alpha / 2)^{\lceil 1 / \epsilon\rceil}$, and hence $1-\alpha<(1-\delta)^{m}$.

Let $\mathcal{C}$ be the multiplicity code of order $s$ evaluations of degree $d$ polynomials in $m$ variables over $\mathbb{F}_{q}$. Observe that $\mathcal{C}$ has length $n$ and is over an alphabet of size $q^{\left(\begin{array}{c}m+s-1 \\ m\end{array}\right)}=$ $n^{O(1)}$. By Lemma $9, \mathcal{C}$ has distance $\delta$ and rate at least $(1-$ $\left.\frac{m^{2}}{s}\right) \cdot(1-\delta)^{m}>1-\alpha$. By Theorem $10, \mathcal{C}$ can be locally selfcorrected from $\delta / 10$-fraction errors using $O\left(n^{1 / m}\right)=O\left(n^{\epsilon}\right)$ queries. This completes the proof of Theorem 3.

Finally, we complete the proof of Theorem 4, by concatenating suitable multiplicity codes with good linear codes over small alphabets.

Proof of Theorem 4: Set $\alpha_{1}=\alpha / 2$ and $\epsilon_{1}=\epsilon / 2$. As in the proof of Theorem 3 , there are constants $m$ and $s$ such that for every prime power $q$, there is a multiplicity code with length $n_{1}=q^{m}$, rate $1-\alpha_{1}$, distance $\delta_{1} \geq \epsilon_{1} \alpha_{1} / 2$, over an alphabet $\Sigma_{1}$ of size $q^{\left(\begin{array}{c}m+s-1 \\ m\end{array}\right)}$, and locally self-correctable from $\delta_{1} / 10$ with $O\left(n_{1}^{\epsilon_{1}}\right)$ queries. We will take such codes $\mathcal{C}_{1}$ where $q=p^{t}$ for integers $t>0$.

We now pick another code $\mathcal{C}_{2}$ of length $\left(\begin{array}{c}m+s-1 \\ m\end{array}\right) \cdot t$ that is $\mathbb{F}_{p}$-linear and has rate $1-\alpha_{1}$ and use it to encode the symbols of $\mathcal{C}_{1}$. The resulting concatenated code $\mathcal{C}$ is $\mathbb{F}_{p}$-linear (this follows from the linearity of $\mathcal{C}_{2}$ and the "pseudo-linearity" of $\mathcal{C}_{1}$ coming from Equation (2)), and has distance $\delta$ and rate $R$ that are at least the products of the corresponding parameters of $\mathcal{C}_{1}$ and $\mathcal{C}_{2}$. In particular, if we take $\mathcal{C}_{2}$ to be a code of constant distance $\delta_{2}>0\left(\mathcal{C}_{2}\right.$ can even be taken to be efficiently constructible, and such that there are efficient error-correction algorithms for decoding upto half the minimum distance), then $\mathcal{C}$ has length $n=q^{m} \cdot\left(\begin{array}{c}m+s-1 \\ m\end{array}\right) \cdot t \cdot \frac{1}{1-\alpha_{1}}$, rate at least $1-\alpha$ and constant (as $n$ grows) distance $\delta>0$.

We now argue that the code $\mathcal{C}$ is locally self-correctable. To locally self-correct some coordinate of a codeword of $\mathcal{C}$ given access to a corrupted codeword of $\mathcal{C}$, we first run the local self-corrector for $\mathcal{C}_{1}$ to decode the coordinate of $\mathcal{C}_{1}$ that contains that coordinate of $\mathcal{C}$. Whenever this local self-corrector wants to query a certain coordinate of $\mathcal{C}_{1}$, we recover that symbol by decoding the corresponding codeword of $\mathcal{C}_{2}$ (if we only care about query complexity, this can be done by brute force; if we are interested in having sublinear running time, then $\mathcal{C}_{2}$ should be chosen so that this step can be done in time polynomial in the length of $\mathcal{C}_{2}$ ). The query complexity of the local self-corrector for $\mathcal{C}$ is clearly $O\left(n^{\epsilon_{1}} \log n\right)=O\left(n^{\epsilon}\right)$. It remains to note that in case the total fraction of errors is below $\delta / 20$, all but $\delta_{1} / 10$ fraction of the $\mathcal{C}_{2}$ blocks will have $<\delta_{2} / 2$-fraction errors, and can be correctly recovered by the decoder for $\mathcal{C}_{2}$. Thus the local self-corrector for $\mathcal{C}_{1}$ will run correctly, and this yields the desired corrected coordinate of $\mathcal{C}$.

\section{LOCAL SELF-CORRECTION OF MUL- TIPLICITY CODES}

In this section, we prove that multiplicity codes are locally self-correctable.

Suppose we are dealing with the multiplicity code of order $s$ evaluations of degree $d$ polynomials in $m$ variables over $\mathbb{F}_{q}$. Let $\Sigma$ be the alphabet for this code. Let $r: \mathbb{F}_{q}^{m} \rightarrow \Sigma$ be a received word. Suppose $P$ is a polynomial over $\mathbb{F}_{q}$ in $m$ variables of degree at most $d$ such that $\Delta\left(r, \operatorname{Enc}_{s, d, m, q}(P)\right)$ is small. Let $\mathbf{a} \in \mathbb{F}_{q}^{m}$. Let us show how to locally recover $P^{(<s)}(\mathbf{a})$ given oracle access to $r$.

As indicated in the introduction, the idea is to pick many random lines containing a, and to consider the restriction of $r$ to those lines. With high probability over a random direction $\mathbf{b} \in \mathbb{F}_{q}^{m} \backslash\{0\}$, by looking at the restriction of $r$ to the line $\mathbf{a}+\mathbf{b} T$ and "decoding" it, we will be able to recover the univariate polynomial $P(\mathbf{a}+\mathbf{b} T)$. Knowing this univariate polynomial will tell us a certain linear combination of the various derivatives of $P$ at $\mathbf{a},\left\langle P^{(\mathbf{i})}(\mathbf{a})\right\rangle_{\mathrm{wt}(\mathbf{i})<s}$. Combining this information for various directions $\mathbf{b}$, we will know a system of various linear combinations of the numbers $\left\langle P^{(\mathbf{i})}(\mathbf{a})\right\rangle_{\mathrm{wt}(\mathbf{i})<s}$. Solving this linear system, we get $P^{(\mathbf{i})}(\mathbf{a})$ for each $\mathbf{i}$, as desired.

To implement this strategy we need to relate the derivatives of the restriction of a multivariate polynomial $P$ to a line to the derivatives of $P$ itself. Fix $\mathbf{a}, \mathbf{b} \in \mathbb{F}_{q}^{n}$, and consider the polynomial $Q(T)=P(\mathbf{a}+\mathbf{b} T)$.

- The relationship of $Q(T)$ with the derivatives of $P$ at a: By the definition of Hasse derivatives,

$$
Q(T)=\sum_{\mathbf{i}} P^{(\mathbf{i})}(\mathbf{a}) \mathbf{b}^{\mathbf{i}} T^{\mathrm{wt}(\mathbf{i})} .
$$

Grouping terms, we see that:

$$
\sum_{\mathbf{i} \mid \operatorname{wt}(\mathbf{i})=e} P^{(\mathbf{i})}(\mathbf{a}) \mathbf{b}^{\mathbf{i}}=\text { coefficient of } T^{e} \text { in } Q(T) .
$$

- The relationship of the derivatives of $Q$ at $t$ with the derivatives of $P$ at $\mathbf{a}+t \mathbf{b}$ : Let $t \in \mathbb{F}_{q}$. By the definition of Hasse derivatives, we get the following two identities:

$$
\begin{gathered}
P(\mathbf{a}+\mathbf{b}(t+R))=Q(t+R)=\sum_{j} Q^{(j)}(t) R^{j} . \\
P(\mathbf{a}+\mathbf{b}(t+R))=\sum_{\mathbf{i}} P^{(\mathbf{i})}(\mathbf{a}+\mathbf{b} t)(\mathbf{b} R)^{\mathbf{i}} .
\end{gathered}
$$


Thus,

$$
Q^{(j)}(t)=\sum_{\mathbf{i} \mid \mathbf{w t}(\mathbf{i})=j} P^{(\mathbf{i})}(\mathbf{a}+\mathbf{b} t) \mathbf{b}^{\mathbf{i}} .
$$

In particular, $Q^{(j)}(t)$ is simply a linear combination of the various $P^{(\mathbf{i})}(\mathbf{a}+\mathbf{b} t)$ (over different $\mathbf{i}$ ).

We are now in a position to describe our decoding algorithm. Before describing the main local self-correction algorithm for correcting from $\Omega(\delta)$-fraction errors, we describe a simpler version of the algorithm which corrects from a much smaller fraction of errors. The analysis of this algorithm will contain many of the ideas. In the description of both algorithms, the query-efficiency will be clear, and we do not comment on how to make them run time-efficiently. In Section 4.3 , we show how the various steps of the algorithms can be made to run in a time-efficient manner as well.

\subsection{Simplified error-correction from few er- rors}

Simplified Local Self-correction Algorithm

Input: received word $r: \mathbb{F}_{q}^{m} \rightarrow \Sigma$, point $\mathbf{a} \in \mathbb{F}_{q}^{m}$. We are trying to recover $P^{(<s)}(\mathbf{a})$, where $P(\mathbf{X})$ is such that $\operatorname{Enc}_{s, d, m, q}(P)$ is close to $r$. Abusing notation, we will write $r^{(\mathbf{i})}(\mathbf{a})$ when we mean the $\mathbf{i}$ coordinate of $r(\mathbf{a})$.

1. Pick a set $B$ of directions: Choose $B \subseteq \mathbb{F}_{q}^{m} \backslash\{0\}$, a uniformly random subset of size $w=\left(\begin{array}{c}m+s-1 \\ m\end{array}\right)$.

2. Recover $P(\mathbf{a}+\mathbf{b} T)$ for directions $\mathbf{b} \in B$ : For each $\mathbf{b} \in B$, consider the function $\ell_{\mathbf{b}}: \mathbb{F}_{q} \rightarrow \mathbb{F}_{q}^{s}$ given by

$$
\left(\ell_{\mathbf{b}}(t)\right)_{j}=\sum_{\mathbf{i} \mid \mathbf{w t}(\mathbf{i})=j} r^{(\mathbf{i})}(\mathbf{a}+\mathbf{b} t) \mathbf{b}^{\mathbf{i}} .
$$

Find the polynomial $Q_{\mathbf{b}}(T) \in \mathbb{F}_{q}[T]$ of degree at most $d$ (if any), such that $\Delta\left(\operatorname{Enc}_{s, d, 1, q}\left(Q_{\mathbf{b}}\right), \ell_{\mathbf{b}}\right)<\delta / 2$.

3. Solve a linear system to recover $P^{(<s)}(a)$ : For each $e$ with $0 \leq e<s$, consider the following sys-

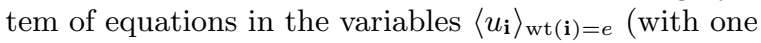
equation for each $\mathbf{b} \in B$ ):

$$
\sum_{\mathbf{i} \mid \mathbf{w t}(\mathbf{i})=e} \mathbf{b}^{\mathbf{i}} u_{\mathbf{i}}=\text { coefficient of } T^{e} \text { in } Q_{\mathbf{b}}(T) .
$$

Find all $\left\langle u_{\mathbf{i}}\right\rangle_{\mathrm{wt}(\mathbf{i})=e}$ which satisfy at all these equations. If there are 0 or $>1$ solutions, output FAIL.

\section{Output the vector $\left\langle u_{\mathbf{i}}\right\rangle_{\mathrm{wt}(\mathbf{i})<s}$.}

Due to lack of space, we will only sketch the analysis of this simplified algorithm. We claim that the above algorithm is a local self-corrector from a $\frac{\delta}{100\left(\frac{m}{m+s-1}\right)}$-fraction of errors. Fix a received word $r: \mathbb{F}_{q}^{m} \rightarrow \Sigma$ and $\mathbf{a} \in \mathbb{F}_{q}^{m}$. Let $P\left(X_{1}, \ldots, X_{m}\right)$ be a polynomial such that $\Delta\left(\operatorname{Enc}_{s, d, m, q}(P), r\right)<$ $\frac{\delta}{100\left(\begin{array}{c}m+s-1 \\ m\end{array}\right)}$. We will call the set of points where $r$ and $\operatorname{Enc}_{s, d, m, q}(P)$ differ the "errors".

The proof has 3 main steps:

Step 1: All the $\mathbf{b} \in B$ are "good". The first step shows that with probability at least 0.9 over the choice of $B$, all the $\mathbf{b} \in B$ will be such that the line through $\mathbf{a}$ in direction b has fewer than $\delta / 2$ errors on it.

Step 2: $Q_{\mathbf{b}}(T)=P(\mathbf{a}+\mathbf{b} T)$ for each $\mathbf{b} \in B$. The next step shows that $Q_{\mathbf{b}}(T)$ is "what it should be".
Step 3: $u_{\mathbf{i}}=P^{(\mathbf{i})}(\mathbf{a})$ for each i. The final step shows, via Equation (3), that the $u_{\mathbf{i}}$ are "what they should be", and furthermore that with probability at least 0.9 over $B$, this is the unique solution to Equation (5).

Overall, with probability at least 0.8 , the algorithm will output $P^{(\mathbf{i})}(\mathbf{a})$, as desired.

\subsection{Error-correction from $\Omega(\delta)$-fraction errors}

We now come to the main local self-correcting algorithm and the proof of Theorem 10. As above, to decode at a point $\mathbf{a}$, we will pick several lines $\mathbf{a}+\mathbf{b} T$ through $\mathbf{a}$, and try to recover the univariate polynomial $P(\mathbf{a}+\mathbf{b} T)$. However, unlike the above algorithm, we will not count on the event that all these lines have less than $\delta / 2$-fraction errors. Instead, we will pick a larger number of lines than the bareminimum required for the next step, and hope that most (but not necessarily all) of these lines will have fewer than $\delta / 2$-fraction errors. Counting on this weakened event allows us to self-correct from a significantly larger fraction of errors. To compensate for the weakening, we will need to make the next step of the algorithm, that of solving a linear system, more robust; we will have to solve a noisy system of linear equations.

Let us elaborate on the method by which we pick the lines in the new algorithm. In the previous algorithm, we picked exactly $\left(\begin{array}{c}m+s-1 \\ m\end{array}\right)$ random lines through a and used them to decode from $\Omega\left(\frac{\delta}{\left(\frac{m+s-1}{m}\right)}\right)$-fraction errors. By picking a larger number of lines, we can decode all the way up to $\Omega(\delta)$-fraction errors. There are several ways of picking this larger number of lines. One way is to pick $\Theta\left(\left(\begin{array}{c}m+s-1 \\ m\end{array}\right)\right)$ independent and uniformly random lines through the point a. The algorithm we present below picks these lines differently; the directions of these lines will come from a random affinely transformed grid. This way of choosing lines admits a simpler analysis, and the noisy system of linear equations that we end up needing to solve turns becomes an instance of the noisy polynomial interpolation problem on a grid, for which time-efficient algorithms are known.

Main Local Self-Correction Algorithm:

Input: received word $r: \mathbb{F}_{q}^{m} \rightarrow \Sigma$, point $\mathbf{a} \in \mathbb{F}_{q}^{m}$. Abusing notation again, we will write $r^{(\mathbf{i})}(\mathbf{a})$ when we mean the $\mathbf{i}$ coordinate of $r(\mathbf{a})$.

1. Pick a set $B$ of directions: Pick $\mathbf{z}, \mathbf{y}_{1}, \mathbf{y}_{2}, \ldots \mathbf{y}_{m} \in$ $\mathbb{F}_{q}^{m}$ independently and uniformly at random. Let $S \subset$ $\mathbb{F}_{q}$ be any set of size $5(s+1)$. Let $B=\left\{\mathbf{z}+\sum_{i=1}^{m} \alpha_{i} \mathbf{y}_{i} \mid\right.$ $\left.\alpha_{i} \in S\right\}$.

2. Recover $P(\mathbf{a}+\mathbf{b} T)$ for directions $\mathbf{b} \in B$ : For each $\mathbf{b} \in B$, consider the function $\ell_{\mathbf{b}}: \mathbb{F}_{q} \rightarrow \mathbb{F}_{q}^{s}$ given by

$$
\left(\ell_{\mathbf{b}}(t)\right)_{j}=\sum_{\mathbf{i} \mid \mathbf{w t}(\mathbf{i})=j} r^{(\mathbf{i})}(\mathbf{a}+\mathbf{b} t) \mathbf{b}^{\mathbf{i}} .
$$

Find the polynomial $Q_{\mathbf{b}}(T) \in \mathbb{F}_{q}[T]$ of degree at most $d$ (if any), such that $\Delta\left(\operatorname{Enc}_{s, d, 1, q}\left(Q_{\mathbf{b}}\right), \ell_{\mathbf{b}}\right)<\delta / 2$.

3. Solve a noisy linear system to recover $P^{(<s)}(a)$ : For each $e$ with $0 \leq e<s$, consider the following system of equations in the variables $\left\langle u_{\mathbf{i}}\right\rangle_{\mathrm{wt}}(\mathbf{i})=e$ (with one equation for each $\mathbf{b} \in B$ ):

$$
\sum_{\mathbf{i} \mid \mathbf{w t}(\mathbf{i})=e} \mathbf{b}^{\mathbf{i}} u_{\mathbf{i}}=\text { coeff of } T^{e} \text { in } Q_{\mathbf{b}}(T) .
$$


Find all $\left\langle u_{\mathbf{i}}\right\rangle_{\mathrm{wt}(\mathbf{i})=e}$ which satisfy at least $3 / 5$ of these equations. If there are 0 or $>1$ solutions, output FAIL.

\section{Output the vector $\left\langle u_{\mathbf{i}}\right\rangle_{\mathrm{wt}(\mathbf{i})<s}$.}

We now proceed to analyze the above algorithm (and thus complete the proof of Theorem 10).

Proof of Theorem 10: For $m=1$ the theorem is trivial, and so we assume $m \geq 2$. Recall that we have $q \geq 10 m$, $q \geq \frac{d+6}{s}$ (so that $q \geq \frac{6}{\delta}$ ) and that $q \geq 5(s+1$ ).

We will show that the above algorithm is a local selfcorrector from a $\frac{\delta}{10}$-fraction of errors. Fix a received word $r: \mathbb{F}_{q}^{m} \rightarrow \Sigma$ and $\mathbf{a} \in \mathbb{F}_{q}^{m}$. Let $P\left(X_{1}, \ldots, X_{m}\right)$ be a polynomial such that $\Delta\left(\operatorname{Enc}_{s, d, m, q}(P), r\right)<\frac{\delta}{10}$. We will call the set of points where $r$ and $\operatorname{Enc}_{s, d, m, q}(P)$ differ the "errors".

Step 1: Many b $\in B$ are "good". For $\mathbf{b} \in \mathbb{F}_{q}^{m} \backslash\{0\}$, we will be interested in the fraction of errors on the line $\left\{\mathbf{a}+t \mathbf{b} \mid t \in \mathbb{F}_{q}\right\}$ through $\mathbf{a}$ in direction $\mathbf{b}$. Since these lines cover $\mathbb{F}_{q}^{m} \backslash\{\mathbf{a}\}$ uniformly, we can conclude that at least $\frac{2}{3}$ of $\mathbf{b} \in \mathbb{F}_{q}^{m}$ are such that the line $\left\{\mathbf{a}+t \mathbf{b} \mid t \in \mathbb{F}_{q}\right\}$ has a fraction of errors which is less than $\left(\frac{\delta}{3}+\frac{1}{q}\right)<\frac{\delta}{2}$. We call these directions $\mathbf{b}$ good. In the claim below, we show that the set $B$ samples the set of good directions well.

Claim 11. Let $m$ be a positive integer, and let $S \subset \mathbb{F}_{q}$ be any set such that $|S|^{m} \geq 50$. Pick $\mathbf{z}, \mathbf{y}_{1}, \mathbf{y}_{2}, \ldots \mathbf{y}_{m} \in$ $\mathbb{F}_{q}^{m}$ independently and uniformly at random. Let $B=\{\mathbf{z}+$ $\left.\sum_{i=1}^{m} \alpha_{i} \mathbf{y}_{i} \mid \alpha_{i} \in S\right\}$. Then for every set $E \subseteq \mathbb{F}_{q}^{m}$ of size at least $2 q^{m} / 3$, the probability that fewer than $3 / 5$ of the points of $B$ lie in $E$ is at most 0.1 .

Proof. The claim follows from a standard application of Chebyshev's inequality, using the fact that the collection of random variables $\left\langle\mathbf{z}+\sum_{i=1}^{m} \alpha_{i} \mathbf{y}_{i}\right\rangle_{\left(\alpha_{1}, \ldots, \alpha_{m}\right) \in S^{m}}$ is pairwise independent.

Hence (recall that we have $m \geq 2$, and so $(5(s+1))^{m}>$ 50 ), with probability at least 0.9 over the choice of $B, 3 / 5$ fraction of the $\mathbf{b} \in B$ will be good.

Step 2: $Q_{\mathbf{b}}(T)=P(\mathbf{a}+\mathbf{b} T)$ for each good $\mathbf{b} \in B$. By Equation (4), for each good $\mathbf{b} \in B$, the corresponding function $\ell_{\mathbf{b}}$ will be such that $\Delta\left(\mathrm{Enc}_{s, d, 1, q}(P(\mathbf{a}+\mathbf{b} T)), \ell_{\mathbf{b}}\right)<\delta / 2$. Thus for each good $\mathbf{b}$, the algorithm will find $Q_{\mathbf{b}}(T)=P(\mathbf{a}+$ $\mathbf{b} T$ ). (Note that at most one polynomial $Q(T)$ of degree at most $d$ has the property that $\Delta\left(\operatorname{Enc}_{s, d, 1, q}(Q), \ell_{\mathbf{b}}\right)<\delta / 2$. This is because for distinct $Q(T), Q^{\prime}(T)$ of degree at most $d$, Lemma 9 implies that $\Delta\left(\operatorname{Enc}_{s, d, 1, q}(Q), \operatorname{Enc}_{s, d, 1, q}\left(Q^{\prime}\right)\right) \geq \delta$.) Step 3: $u_{\mathbf{i}}=P^{(\mathbf{i})}(\mathbf{a})$ for each i. Since $Q_{\mathbf{b}}(T)=P(\mathbf{a}+\mathbf{b} T)$ for each good $\mathbf{b} \in B$, Equation (3) now implies that with probability 0.9 , for each $0 \leq e<s$, the vector $\left\langle u_{\mathbf{i}}\right\rangle_{\mathrm{wt}(\mathbf{i})=e}$ with $u_{\mathbf{i}}=P^{(\mathbf{i})}(\mathbf{a})$ will satisfy at least $3 / 5$ of the equations in the system (6).

Finally, we observe that this solution $u_{\mathbf{i}}$ is unique with probability at least 0.9 . Indeed, with probability at least 0.9 over the choice of $B$, the elements $\mathbf{y}_{1}, \ldots, \mathbf{y}_{m}$ will be linearly independent over $\mathbb{F}_{q}^{m}$ (since $q \geq 10 \mathrm{~m}$ ). In this case, there is an $\mathbb{F}_{q}$-linear map which gives a bijection between $S^{m}$ and $B$. Via this linear map, we get a degree preserving correspondence between polynomials evaluated on $S^{m}$ and polynomials evaluated on $B$. Now by Lemma 7 (and recalling that $|S|=5(s+1)$ ), there is no nonzero polynomial of degree $<s$ that vanishes on more that $1 / 5$-fraction of the points of $S^{m}$. Hence no nonzero polynomial of degree $<s$ vanishes on more that $1 / 5$-fraction of the points of $B$.
Hence for each $e$, the vector $\left\langle u_{\mathbf{i}}\right\rangle_{\mathrm{wt}(\mathbf{i})=e}$ that satisfies $3 / 5$ of the equations in the system (6) is unique; if not, then the difference $\left\langle u_{\mathbf{i}}-u_{\mathbf{i}}^{\prime}\right\rangle_{\mathrm{wt}(\mathbf{i})=e}$ of two such vectors $\left\langle u_{\mathbf{i}}\right\rangle_{\mathrm{wt}(\mathbf{i})=e},\left\langle u_{\mathbf{i}}^{\prime}\right\rangle_{\mathrm{wt}(\mathbf{i})=e}$ will be the coefficients of a polynomial of degree $<s$ that vanishes on at least $1 / 5$ fraction of $B$; for any $\mathbf{b} \in B$ such that both $\left\langle u_{\mathbf{i}}\right\rangle_{\mathrm{wt}(\mathbf{i})=e}$ and $\left\langle u_{\mathbf{i}}^{\prime}\right\rangle_{\mathrm{wt}(\mathbf{i})=e}$ satisfy the equation (6), we have: $\sum_{\mathbf{i} \mid \operatorname{wt}(\mathbf{i})=e}\left(u_{\mathbf{i}}-u_{\mathbf{i}}^{\prime}\right)\left(\mathbf{b}^{\mathbf{i}}\right)=0$, contradicting the fact that there is no nonzero polynomial of degree $<s$ that vanishes on more that $1 / 5$-fraction of the points of $B$.

Overall, with probability at least 0.8 , the algorithm will output $P^{(\mathbf{i})}(\mathbf{a})$, as desired.

This completes the proof of Theorem 10 .

\subsection{Running time}

In this section, we will see how the above local self-correction algorithms can be made to run efficiently, in time polynomial in the query complexity.

There are two key steps which we need to elaborate on. The first step is the search for the univariate polynomial $Q_{\mathbf{b}}(T)$. Here the problem boils down to the problem of decoding univariate multiplicity codes up to half their minimum distance. The second step we will elaborate on is solving the noisy linear system of equations. This will reduce to an instance of decoding Reed-Muller codes.

Decoding univariate multiplicity codes. We deal with the first step first, that of decoding univariate multiplicity codes. Explicitly, let $\Sigma=\mathbb{F}_{q}^{s}$, we have a function $\ell: \mathbb{F}_{q} \rightarrow \Sigma$, and we want to find the univariate polynomial $Q(T) \in \mathbb{F}_{q}[T]$ of degree at most $d$ such that $\Delta\left(\ell, \operatorname{Enc}_{s, d, 1, q}(Q)\right)<(1-$ $d / s q) / 2=\delta / 2$. Abusing notation again, we let $\ell^{(i)}: \mathbb{F}_{q} \rightarrow$ $\mathbb{F}_{q}$ be the function which equals the $i$-coordinate of $\ell$, for $0 \leq i<s$.

Univariate multiplicity codes are instances of "ideal errorcorrecting codes" [17, 34]. Formally defining ideal errorcorrecting codes will take us too far afield; we will content ourselves with instantiating the known algorithm for decoding ideal error-correcting codes in this case, and explicitly writing out the resulting efficient algorithm for decoding univariate multiplicity codes. All these algorithms are extensions of the beautiful Berlekamp-Welch algorithm for decoding Reed-Solomon codes.

Let $Q(T)$ be a polynomial of degree at most $d$ such that $\Delta\left(\ell, \operatorname{Enc}_{s, d, 1, q}(Q)\right)<\delta / 2$. Our underlying goal is to search for polynomials $E(T), N(T)$ such that $N(T)=E(T) Q(T)$ (and so we obtain $Q(T)$ as $N(T) / E(T)$ ). By the product rule for Hasse derivatives (which states that $\left(P_{1} \cdot P_{2}\right)^{(i)}(T)=$ $\sum_{j=0}^{i} P_{1}^{(j)}(T) P_{2}^{(i-j)}(T)$, see [19]), such polynomials $E(T)$, $N(T)$ will also satisfy the equalities

$$
\begin{gathered}
N^{(1)}(T)=(E \cdot Q)^{(1)}(T)=E(T) Q^{(1)}(T)+E^{(1)}(T) Q(T), \\
\ldots \\
N^{(s-1)}(T)=(E \cdot Q)^{(s-1)}(T)=\sum_{i=0}^{s-1} E^{(i)}(T) Q^{(s-1-i)}(T)
\end{gathered}
$$

This motivates the following algorithm.

- Search for nonzero polynomials $E(T), N(T)$ of degrees at most $(s q-d) / 2,(s q+d) / 2$ respectively such that 
for each $x \in \mathbb{F}_{q}$, we have the following equations:

$$
\begin{aligned}
N(x) & =E(x) \ell^{(0)}(x) \\
N^{(1)}(x) & =E(x) \ell^{(1)}(x)+E^{(1)}(x) \ell^{(0)}(x) \\
& \ldots \\
N^{(s-1)}(x) & =\sum_{i=0}^{s-1} E^{(i)}(x) \ell^{(s-1-i)}(x)
\end{aligned}
$$

This is a collection of $s q$ homogeneous linear equations in $(s q-d) / 2+1+(s q+d) / 2+1>s q$ unknowns (the coefficients of $E$ and $N)$. Thus a nonzero solution $E(T), N(T)$ exists. Take any such nonzero solution.

- Given $E, N$ as above, output $N / E$.

To see correctness, take any $Q$ such that $\Delta\left(\ell, \operatorname{Enc}_{s, d, m, q}(Q)\right)<$ $\delta / 2$. Observe that for any $x$ where $\ell(x)=\operatorname{Enc}_{s, d, m, q}(Q)(x)$, the system of equations ( 7$)$ is satisfied at $T=x$, and hence the polynomial $N(T)-E(T) Q(T)$ has a root with multiplicity $s$ at $x$. Thus $\sum_{x \in \mathbb{F}_{q}} \operatorname{mult}(N-E Q, x)>(1-\delta / 2) s q=$ $(s q+d) / 2$. Since $\operatorname{deg}(N-E Q) \leq(s q+d) / 2$, we conclude that the polynomial $N-E Q$ must be identically 0 , and hence $Q(T)=N(T) / E(T)$, as desired (here we used the fact that $E, N$ are not both identically 0$)$. In particular $E \mid N$, and $N / E$ is a polynomial.

Solving the noisy system. We now show how to solve the noisy system of linear equations efficiently. For $m$ and $s$ constant, this is a system of constantly many $\left(O(s)^{\mathrm{m}}\right)$ linear equations over $\mathbb{F}_{q}$, and hence by running over all subsystems consisting of $3 / 5$-fraction of these equations, and solving that subsystem of linear equations exactly, we can solve the noisy system in time $\exp \left(s^{m}\right) \cdot \operatorname{poly} \log q$.

This is somewhat unsatisfactory. We point out some special situations where solving these noisy linear equations can be done in (optimal) time poly $\left(s^{m}, \log q\right)$. Observe that our task is of the form: "Given a function $r: B \rightarrow \mathbb{F}_{q}$, find all polynomials $R\left(X_{1}, \ldots, X_{m}\right) \in \mathbb{F}_{q}\left[X_{1}, \ldots, X_{m}\right]$ of degree $<s$ such that $R(\mathbf{b})=r(\mathbf{b})$ for at least $\alpha$-fraction of the $\mathbf{b} \in B$ ". Thus, this is a problem of noisy polynomial interpolation. As observed in Step 3 of the analysis of the main local self-correction algorithm, there is a linear map which puts $S^{m}$ and $B$ in bijection. This linear map gives rise to a degree-preserving correspondence between polynomials evaluated on $S^{m}$ and polynomials evaluated on $B$. Thus, our task of doing noisy polynomial interpolation (for polynomials of degree $<s$ ) on $B$ reduces to noisy polynomial interpolation (for polynomials of degree $<s$ ) on $S^{m}$. This brings us into familiar territory. For certain fields $\mathbb{F}_{q}$, and certain choices of the set $S$, there are known efficient algorithms for doing this. In particular, if $q$ is a power of $p$, and $S$ is an $\mathbb{F}_{p}$-subspace of $\mathbb{F}_{q}$, given a function $r: S^{m} \rightarrow \mathbb{F}_{q}$, one can recover the unique degree $<s$ polynomial $R$ that agrees with $r$ in at least $(1+s / q) / 2$-fraction of the points of $S^{m}$ in time poly $\left(|S|^{m}, \log q\right)$. If $q>4 s$, then this fraction is at most $3 / 5$, and this suffices for application to the local self-correcting algorithm of the previous section.

\section{DISCUSSION}

Multiplicity codes are a natural and basic family of codes. Their similarity to multivariate polynomial codes endows them with a useful geometric structure, and their better rate/distance tradeoff allows them to carry that usefulness into new combinatorial regimes.
There are a number of questions related to multiplicity codes that invite further exploration.

- Because of their high rate, multiplicity codes seem to have potential for use in practice. It will be very interesting to see if these codes can find use in practical settings.

Here is a concrete setting given by multiplicity codes. Suppose we have a storage medium which stores data in 150 bit blocks. Then, 720 million blocks of data can be encoded at rate $\approx 0.72$ to become $\mathrm{a} \approx 1$ billion block long codeword, such that even if an arbitrary 10 million blocks of the codeword get corrupted, one can recover with probability $>0.95$ any desired block of the original data by just looking at 640,000 blocks of the corrupted codeword. Earlier, codes of this rate would have required one to read and process at least $\approx 720$ million blocks of the codeword to recover 1 block of original data.

- For every $\epsilon>0$ multiplicity codes yield positive-rate $O\left(n^{\epsilon}\right)$-query LDCs tolerating a constant fraction of errors. It is a very interesting problem to see if there exist positive rate LDCs with even lower query complexity. The only known lower bound on the query complexity in this regime is $\Omega(\log n)$.

- Finally, it would be interesting to see if multiplicity codes can be useful in the various other settings where multivariate polynomial codes have proven useful.

\section{REFERENCES}

[1] S. Arora, C. Lund, R. Motwani, M. Sudan, and M. Szegedy. Proof verification and the hardness of approximation problems. Journal of the ACM, 45(3):501-555, May 1998.

[2] S. Arora and S. Safra. Probabilistic checking of proofs: A new characterization of NP. Journal of the ACM, 45(1):70-122, January 1998.

[3] S. Arora and M. Sudan. Improved low-degree testing and its applications. Combinatorica, 23:365-426, 2003.

[4] L. Babai, L. Fortnow, L. Levin, and M. Szegedy. Checking computations in polylogarithmic time. In 23rd ACM Symposium on Theory of Computing (STOC), pages 21-31, 1991.

[5] L. Babai, L. Fortnow, and C. Lund. Non-deterministic exponential time has two-prover interactive protocols. Computational Complexity, 1(1):3-40, 1991.

[6] L. Babai, L. Fortnow, N. Nisan, and A. Wigderson. BPP has subexponential time simulations unless EXPTIME has publishable proofs. Computational Complexity, 3:307-318, 1993.

[7] D. Beaver and J. Feigenbaum. Hiding instances in multioracle queries. In 7 th International Symposium on Theoretical Aspects of Computer Science (STACS), volume 415 of Lecture Notes in Computer Science, pages 37-48. Springer, Berlin, Heidelberg, 1990.

[8] A. Ben-Aroya, K. Efremenko, and A. Ta-Shma. Local list decoding with a constant number of queries. In 51st IEEE Symposium on Foundations of Computer Science (FOCS), 2010.

[9] Y. M. Chee, T. Feng, S. Ling, H. Wang, and L. F. Zhang. Query-efficient locally decodable codes of subexponential length. Arxiv 1008.1617, 2010. 
[10] A. Deshpande, R. Jain, T. Kavitha, S. Lokam, and J. Radhakrishnan. Better lower bounds for locally decodable codes. In 20th IEEE Computational Complexity Conference (CCC), pages 184-193, 2002.

[11] Z. Dvir. On matrix rigidity and locally self-correctable codes. In 26th IEEE Computational Complexity Conference (CCC), pages 102-113, 2010.

[12] Z. Dvir, P. Gopalan, and S. Yekhanin. Matching vector codes. In 51st IEEE Symposium on Foundations of Computer Science (FOCS), 2010.

[13] Z. Dvir, S. Kopparty, S. Saraf, and M. Sudan. Extensions to the method of multiplicities, with applications to Kakeya sets and mergers. In 50th IEEE Symposium on Foundations of Computer Science (FOCS), pages 181-190, 2009.

[14] K. Efremenko. 3-query locally decodable codes of subexponential length. In 41st ACM Symposium on Theory of Computing (STOC), pages 39-44, 2009.

[15] O. Goldreich, H. Karloff, L. Schulman, and L. Trevisan. Lower bounds for locally decodable codes and private information retrieval. In 17th IEEE Computational Complexity Conference (CCC), pages 175-183, 2002.

[16] V. Guruswami and A. Rudra. Explicit codes achieving list decoding capacity: Error-correction with optimal redundancy. IEEE Transactions on Information Theory, 54(1):135-150, 2008.

[17] V. Guruswami, A. Sahai, and M. Sudan. Soft-decision decoding of Chinese Remainder codes. In Proceedings of the 41st IEEE Symposium on Foundations of Computer Science, pages 159-168, Redondo Beach, California, 12-14 November 2000.

[18] V. Guruswami and M. Sudan. Improved decoding of Reed-Solomon and algebraic-geometric codes. IEEE Transactions on Information Theory, 45:1757-1767, 1999.

[19] J. W. P. Hirschfeld, G. Korchmaros, and F. Torres. Algebraic Curves over a Finite Field (Princeton Series in Applied Mathematics). Princeton University Press, 2008.

[20] R. Impagliazzo and A. Wigderson. P $=$ BPP if $E$ requires exponential circuits: Derandomizing the XOR Lemma. Proceedings of the 29th Annual ACM Symposium on Theory of Computing, pages 220-229, May 1997.

[21] T. Itoh and Y. Suzuki. New constructions for query-efficient locally decodable codes of subexponential length. IEICE Transactions on Information and Systems, pages 263-270, 2010.

[22] J. Katz and L. Trevisan. On the efficiency of local decoding procedures for error-correcting codes. In 32nd ACM Symposium on Theory of Computing (STOC), pages 80-86, 2000.

[23] I. Kerenidis and R. de Wolf. Exponential lower bound for 2-query locally decodable codes via a quantum argument. Journal of Computer and System Sciences, 69:395-420, 2004.

[24] R. Lipton. Efficient checking of computations. In 7th International Symposium on Theoretical Aspects of Computer Science (STACS), volume 415 of Lecture Notes in Computer Science, pages 207-215. Springer, Berlin, Heidelberg, 1990.
[25] C. Lund, L. Fortnow, H. J. Karloff, and N. Nisan. Algebraic methods for interactive proof systems. Journal of the ACM, 39(4):859-868, October 1992.

[26] K. Obata. Optimal lower bounds for 2-query locally decodable linear codes. In 6th International Workshop on Randomization and Computation (RANDOM), volume 2483 of Lecture Notes in Computer Science, pages 39-50. Springer, Berlin, Heidelberg, 2002.

[27] F. Parvaresh and A. Vardy. Correcting errors beyond the Guruswami-Sudan radius in polynomial time. In 46th IEEE Symposium on Foundations of Computer Science (FOCS), pages 285-294, 2005.

[28] P. Raghavendra. A note on Yekhanin's locally decodable codes. In Electronic Colloquium on Computational Complexity (ECCC), TR07-016, 2007.

[29] I. S. Reed. A class of multiple-error-correcting codes and the decoding scheme. IEEE Transactions on Information Theory, 4:38-49, 1954.

[30] M. Rozenbloom and M. Tsfasman. Codes for the m-metric. Problems Inform. Transmission, 33:45-52, 1997.

[31] S. Saraf and M. Sudan. Improved lower bound on the size of Kakeya sets over finite fields. Analysis and PDE, 2008.

[32] R. Shaltiel and C. Umans. Simple extractors for all min-entropies and a new pseudorandom generator. $J$. ACM, 52(2):172-216, 2005.

[33] A. Shamir. IP = PSPACE. Journal of the ACM, 39(4):869-877, October 1992.

[34] M. Sudan. Ideal error-correcting codes: Unifying algebraic and number-theoretic algorithms. In $A A E C C$, pages 36-45, 2001.

[35] M. Sudan, L. Trevisan, and S. Vadhan. Pseudorandom generators without the XOR lemma. In 39th ACM Symposium on Theory of Computing (STOC), pages 537-546, 1999.

[36] S. Wehner and R. de Wolf. Improved lower bounds for locally decodable codes and private information retrieval. In 32nd International Colloquium on Automata, Languages and Programming (ICALP), volume 3580 of Lecture Notes in Computer Science, pages 1424-1436. Springer, Berlin, Heidelberg, 2005.

[37] D. Woodruff. New lower bounds for general locally decodable codes. In Electronic Colloquium on Computational Complexity (ECCC), TR07-006, 2007.

[38] D. Woodruff and S. Yekhanin. A geometric approach to information theoretic private information retrieval. In 20th IEEE Computational Complexity Conference $(C C C)$, pages 275-284, 2005.

[39] C. Xing. Nonlinear codes from algebraic curves improving the Tsfasman-Vladut-Zink bound. IEEE Transactions on Information Theory, 49(7):1653-1657, 2003.

[40] S. Yekhanin. Towards 3-query locally decodable codes of subexponential length. Journal of the ACM, 55:1-16, 2008.

[41] S. Yekhanin. Locally decodable codes. Foundations and trends in theoretical computer science, 2010. to appear. 